majority of brain atrophy will have taken place, and the measurement of improvement following immunosuppressive treatment will not be possible. Estimation of hemispheric volume loss may be used as a parameter of the destructive disease process during the acute phase. An autoimmune response mediated by cytotoxic $\mathrm{T}$ lymphocytes is proposed as a key mechanism in the etiology of Rassmussen's encephalitis (Bauer J, Bien CG, Lassmann H. see Ped Neur Briefs April 2002;16:27-28).

\title{
ANTI-BASAL GANGLIA ANTIBODIES IN SYDENHAM'S CHOREA
}

The sensitivity and specificity of methods to detect anti-basal ganglia antibodies (ABGA) in Sydenham's chorea were determined in a study at the Institute of Neurology, Queen Square; Great Ormond Street Hospital for Children; Royal Free and University College Hospital Medical School, London, UK; and Federal University of Minas Gerais, Brazil. Samples from 20 patients with acute SC, 16 with persistent SC, control samples from 16 with rheumatic fever (RF), and 11 healthy pediatric volunteers were tested with ELISA and Western immunoblotting (WB) methods to detect ABGA and compared these assays to immunofluorescent (IF) methods. In acute SC, a sensitivity of $95 \%$ and specificity of $93 \%$ were obtained with ABGA ELISA, while WB and IF had a sensitivity of $100 \%$ and specificity of $93 \%$. In persistent SC, ABGA sensitivity was $69 \%$ using WB and $63 \%$ with IF. WB identified 3 common basal ganglia antigens ( $40 \mathrm{kDa}, 45 \mathrm{kDa}$, and $60 \mathrm{kDa})$ in both acute and persistent SC cases. Antibody reactivity to cerebellum, cerebral cortex, or myelin antigen was absent in all groups. WB and IF are recommended for detecting $\mathrm{ABGA}$ in acute and persistent cases of SC. The results support an autoantibody-mediated mechanism in SC. (Church AJ, Cardoso F, Dale RC et al. Anti-basal ganglia antibodies in acute and persistent Sydenham's chorea. Neurology July (2 of 2) 2002;59:227-231). (Reprints: Mr Andrew Church, Neuroimaging Unit, Room 917, Institute of Neurology, Queen Square, London WC1N 3BG, UK).

COMMENT. Anti-basal ganglia antibodies are reported in Sydenham's chorea and pediatric autoimmune neuropsychiatric disorder associated with streptococcus (PANDAS). Pathological studies in SC show basal ganglia involvement. An antibody-mediated mechanism is suggested by the latency of the basal ganglia syndromes that may develop after streptococcal infection. WB and indirect IF are the best methods for detecting ABGA, whereas ELISA may be used to monitor ABGA levels over time and to assess treatment response.

\section{DEVELOPMENTAL DISORDERS}

\section{SIMPLIFIED CLASSIFICATION OF FOCAL CORTICAL DYSPLASIA}

Sections of cortex from 52 of 224 (23\%) patients with cortical dysplasia, operated on for drug-resistant partial epilepsy, were retrospectively re-examined histologically at Niguarda Hospital, and Istituto Nazionale Neurologico 'C. Besta', Milan, Italy. Three subgroups were identified as follows: 1) architectural dysplasia (31 patients) with abnormal cortical lamination and ectopic neurons in white matter; 2) cytoarchitectural dysplasia (6 patients) with altered cortical lamination and giant neurofilament-enriched neurons; and 3) Taylor-type cortical dysplasia (15 patients) with cortical laminar disruption and giant dysmorphic neurons and balloon cells. Group 1 architectural dysplasia cases had a temporal lobe location for the epileptogenic zone, with focal hypoplasia, and a significantly lower seizure frequency than groups 2 and 3. Group 3 Taylor-type dysplasia patients had an extratemporal epileptogenic zone, interictal distinctive stereo-EEG with high 
frequency fast spikes and polyspikes interspersed with fast low amplitude activity, and MRI signal alterations with thickening of cortex, blurred grey-white matter junction and hyperintensity of subcortical white matter. Group 3 Taylortype had the best outcome, with $75 \%$ seizure-free after one year follow-up, compared with $50 \%$ in type 2 cytoarchitectural cases, and $43 \%$ seizure-free in type 1 architectural dysplasia cases. The three-category classification has easily recognized histopathological findings, and clinically homogeneous characteristics. (Tassi L, Colombo N, Garbelli R et al. Focal cortical dysplasia: n europathological subtypes, EEG, neuroimaging and surgical outcome. Brain Auguist 2002;125:1719-1732). (Respond: Robert Spreafico MD PhD, Department of Experimental Neurophysiology and Epileptology, Instituto Nazionale Neurologico 'C. Besta', Via Celoria 11, 20133 Milano, Italy).

COMMENT. In the above series of focal cortical dysplasia, the video or stereo-EEG provided the best delineation of the epileptogenic zone, superior to the MRI. In one-third of cases, the MRI was unremarkable, and in those with MRI evidence of a lesion, the boundaries were ill-defined. Type 3 Taylor-type dysplasia

had a better outcome than the type 1 architectural and type 2 cytoarchitectural dysplasias.

\section{HEREDITARY SUSCEPTIBILITY TO FETAL VALPROATE SYNDROME}

Three families in whom all 7 siblings were affected by fetal valproate syndrome (FVS) are reported from the Department of Medical Genetics, Family Federation of Finland, Helsinki, Finland. The parents of affected children had normal intelligence and were nonconsanguinous. Maternal epilepsy was idiopathic, myoclonus or secondary to arteriovenous malformation. Maternal age ranged from 23 to 34 . Daily valproate dosage ranged from a low of $1000-1200 \mathrm{mg}$ to $2000-2500 \mathrm{mg}$, with serum valproate levels of $41-46$ to $77-101 \mathrm{mg} / \mathrm{L}$. Age at examination of the 7 affected children varied from 1 yr 5 months to 8 years 0 months. Developmental deficit was mild to moderate in all patients. Malformations involved the face, urinary tract, cleft palate, hands (small finger nails) and feet, and inguinal hernias or undescended testis in all. Craniosynostosis (trigonocephaly) occurred in 2, tracheomalacia and ventriculomegaly in 1 , and severe hearing deficit in 1 . No neural tube defects occurred in these patients, only 2 of the mothers having received folic acid, only 1 prior to conception. Neonatal VPA withdrawal symptoms (convulsions, feeding problems or respiratory distess) occurred in 5 infants; these resolved promptly with treatment and did not account for the developmental delay. The occurrence of FVS in all the siblings strongly suggests an hereditary susceptibility to valproate embryopathy. (Malm H, Kajantie E, Kivirikko S et al. Valproate embryopathy in three sets of siblings: Further proof of hereditary susceptibility. Neurology August (2 of 2) 2002;59:630-633). (Reprints: Dr Heli Malm, Teratology Information, Department of Medical Genetics, Family Federation of Finland, PO Box 849, Helsinki, FIN00101, Finland).

COMMENT. Hereditary susceptibility to fetal valproate syndrome (FVS) is suggested by this report of three families with all siblings affected. FVS is characterized by typical facial features (medial deficiency of eyebrows, broad nasal root, anteverted nares, shallow philtrum, and long and thin upper lip), trigonocephaly, developmental delay, neural tube, cardiac and genitourinary malformations, and limb defects. The risk of recurrence in a subsequent pregnancy may be high, indicating avoidance of further VPA therapy. 